\title{
A Novel Method of Minimal Dominating Node Selection and Comparison with Dai and Wu Algorithm for Energy Efficient Routing in Ad hoc Network based on Directional Antenna
}

\author{
Harinandan Tunga \\ Department of Computer Sc. \& Engineering, \\ RCC Institute of Information Technology \\ Kolkata, West Bengal, India
}

\begin{abstract}
Energy consumption is one of the most important performance metrics for wireless ad hoc networks because it directly relates to the operational lifetime of the network. Based on this thought, in this article we will establish an ad-hoc network where the power consumption during transmission can be controlled.

To minimize the total energy consumption, we have adopted a simple network layer/routing model that serves as a benchmark for performance evaluation. Herein we have worked with the mesh topology, and each nodes of the network are substituted using directional antenna. The four directional antennas are used to represent each node. When a node needs to send information to others it only enables its particular sector or sectors through which it is connected to those nodes. We have also replaced the nodes in the network with sectors and found that the total energy consumed by the network is much less. We devised an algorithm to find the average dominating set without varying the radius of the network. Also, we found the minimum dominating set within a graph by varying the number of nodes only i.e. the density of the graphs. After establishing our own set of algorithm to find the minimum dominating set in a network, it is compared with Dai \& Wu's Algorithm .We believe by using such a generic model, with special added advantages, we will succeed in meeting our goal.
\end{abstract}

\section{Keywords}

Connected Dominating Set (CDS), Directional Antenna (DA), Dominating Sector (DS), Energy Efficient Routing (EER), Minimal Dominating Node Selection (MDNS) and Minimal Connected Dominating Set (MCDS).

\section{INTRODUCTION}

Wireless ad hoc network is a network without physical backbone. Herein a communication session is established through single-hop radio transmission if sender and receiver are within transmission range of each other, otherwise intermediate nodes relay packets. Due to independence from central network administration and its ability to support tetherless access to a variety of services, the ad hoc networks are hugely preferred. Energy conservation of the participating nodes is a key point in all issues related to wireless ad hoc or sensor network, especially in case of broadcasting. CDS acts as a virtual backbone to compensate the absence of physical backbone infrastructure. Any node having information to transmit sends it to nearest CDS member which then forwards the message to its destination. Non-CDS nodes may sleep for some time and after waking up will receive anything destined to it from nearest CDS member. Recently, the directional antenna model has been developed [10] for; each antenna improves the quality of individual links and decrease the overall interference. The common directional antenna model, basically involving a NPcomplete approach, involves dividing the transmission range of a node into $\mathrm{K}$ identical sectors, and one or more sectors can be switched on to transmit/receive. Alzoubi and Wan [4] also worked on to construct dominating set first and then make the component connected. Another most feasible algorithm was proposed by $\mathrm{Wu}$ and $\mathrm{Li}$ [13][17] which initially constructs a CDS and then tries to reduce the size. In Ikiz, Ogale and Garg [5] proposed an energy conserving backbone based routing technique for ad hoc networks. Most of the work followed probabilistic approach in the beginning. However, localized deterministic approaches are considered to be better. Shen et al.[21] devised directional versions of probabilistic protocols. Dai and $\mathrm{Wu}[3][10][11][20]$ proposed a deterministic localized routing protocol using directional antennas, where directional self- pruning (DSP) is developed to reduce transmission directions. However, DSP is used for efficient broadcasting and routing, where the source is known. All of the above schemes discussed herein assumes an omnidirectional reception mode.

\section{PROBLEM ANALYSIS}

A wireless ad-hoc network is a decentralized wireless network, implying that it does not rely on a preexisting infrastructure, such as routers in wired networks or access points in managed (infrastructure) wireless networks. Instead routing based on connected dominating set is considered whereby searching space for a route is reduced to nodes in the set. Thereby routing of packets through nodes with low residual energy, to optimize flooding of routing information over the network and to avoid interference and medium collisions. A single node failure in sensor networks is usually unimportant if it does not lead to a loss of sensing and communication coverage; ad-hoc networks, instead, are oriented towards personal communication and the loss of connectivity to any node is significant.

For protocols that belong to the former category, the active communication energy can be reduced by adjusting each node's radio power just enough to reach the receiving node but not more than that. And for latter one each node can save the inactivity energy by switching its mode of operation into sleep/power-down mode or simply turns it off when there is no data to transmit or receive(mainly for individual nodes). Another important approach to optimizing active communication energy is load distribution approach, mainly to balance the energy usage among the nodes and to maximize the network lifetime by avoiding over-utilized nodes while selecting a routing path. To achieve energy efficiency directional antenna for a wireless networks, the devices operating on battery try to pursue the energy efficiency 
heuristically by reducing the energy they consume, while maintaining acceptable performance of certain tasks. Though for multi-hop routing, which is typical for ad hoc and sensor networks, this is not the optimal strategy. There are also other ways to categorize routing algorithms i.e. in terms of broadcast, multicast and unicast. It goes without saying that node failure is very much possible in the wireless network. Hence saving energy when broadcasting in order to recover from the node failure or to re-routing around the failed nodes is essential. Sometimes, shortest path routing is possibly not the best choice from the energy efficiency point of view. With the help of switched beam and steerable beam techniques, antenna systems can now form directional transmission and/or reception.

\section{PROPOSED METHOD DISCU-SSION AND IMPLEMENTA-TION}

As discussed, the aim of our paper is to develop or implement an algorithm for DA based MDNS for EER. Now a detailed overview of our implemented algorithm is shall be provided.

At first, the topology of the entire network has to be ascertained before we proceed with the implementation. Considering multihop wireless network, a network topology is described, a connected graph is used; mesh topology serves as a network. Now for each node, a range has been fixed. Nodes basically behave as routers that discover and maintain routes to other nodes. Depending on the generated graph the dominating set has been made using one hop information for each node. The issues in routing in adhoc network give rise to many tradeoffs that must be addressed. The time varying topology requires that the topology information must be exchanged frequently so that every node is updated with latest information. At the time of determination of dominating set, each node chooses its own dominating node in parallel. No node has the information which node is the dominating set. Multiple thread works concurrently herein. Each node selects the node whose degree is maximum among the neighboring nodes as dominating node of its own. If there is no neighboring node in its one hop information then it selects itself as the dominating set. After determination of dominating node for each hop, the threads are destroyed and the connected dominating set of nodes in the graph is created. In adhoc networks, dynamic reconfigurability and establishment of routes are the most important features and herein we try and demonstrate the viability of our algorithm based on this point.

\subsection{Illustration of How to Find Dominating Set Participating in Transmission}

In this section illustrates how the connected dominating set is generated with an example-

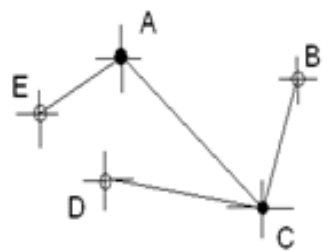

Fig 1: Dominating Set Participating In Transmission

In the above figure we can see that $\{\mathrm{A}, \mathrm{C}\}$ is the dominating set.

The $1^{\text {st }}$ hop of each node is following:

$$
\begin{aligned}
& E=\{A\}, \text { degree }=1 . \\
& A=\{E, C\}, \text { degree }=2 . \\
& C=\{A, B, D\}, \text { degree }=3 .
\end{aligned}
$$

$$
\begin{aligned}
& D=\{C\}, \text { degree }=1 . \\
& B=\{C\}, \text { degree }=1 .
\end{aligned}
$$

So, $\mathrm{E}$ chooses $\mathrm{A}$ as its dominating set. As, $\max \{\mathrm{A}\}=\mathrm{A}$.

A chooses $\mathrm{C}$ as its dominating set. As, $\max \{\mathrm{E}, \mathrm{C}\}=\mathrm{C}$.

$\mathrm{C}$ chooses $\mathrm{A}$ as its dominating set. As, $\max \{\mathrm{A}, \mathrm{B}, \mathrm{D}\}=\mathrm{A}$.

D chooses $\mathrm{C}$ as its dominating set. As, $\max \{\mathrm{C}\}=\mathrm{C}$.

$\mathrm{B}$ chooses $\mathrm{C}$ as its dominating set. As, $\max \{\mathrm{C}\}=\mathrm{C}$.

When using a DA model, each node divides its omnidirectional transmission range into K sectors. Parts of them can be selected to be switched on for transmission. We assume that all nodes use a DA for transmission and an omnidirectional antenna for reception.

To construct a DA we can divide an omnidirectional antenna in $\mathrm{n}$ sectors. So only those sectors of the DA can be activated through which maximum number of nodes of the network can be connected. Therefore the nodes of the dominating set can be substituted by those DAs.

After the directional edges are determined for each forwarding node, the transmission directions can be calculated based on the given number of sectors 4 . We can develop an optimization algorithm to let each node select its DS of the neighbor node to minimize the number of switched-on sectors in the network.

\subsection{Illustration of Sector Optimization of a node}

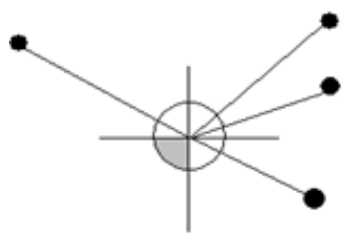

Fig 2: Sector Optimization of a node

So, the number of sector is reduced by 1 . Now, if a complete node is being selected total energy needed for transmitting information would be $\mathrm{P}$. But for this case:

$$
\begin{aligned}
& =3 \times \frac{P}{4} \\
& =0.75 \mathrm{P} \\
& =75 \%
\end{aligned}
$$

So $25 \%$ of energy is reduced and energy efficiency is achieved. Here the gray section of the node shows that this sector is off because it has nothing to transmit to any node.

\subsection{Algorithms of Generating Conn-ected Graph and Generating Connected Dominating Set in a Graph}

Algorithm for Generating Connected Graph: Steps:

1. Generating $\mathrm{n}$ number of nodes in random location.

2. For each node a fixed radius is set.

3. Repeat step 4 for $\mathrm{i} \leftarrow 0$ to $\mathrm{n}-1$ do

4. Now connect those node which are within the radius of node i.

5. End.

Algorithm for Generating CDS in a Graph: Steps:

1. Generating n number of nodes in random location.

2. For each node a fixed radius is set.

3. Repeat step 4 for $\mathrm{i} \leftarrow 0$ to $\mathrm{n}-1$ do 
4. Now connect those node which are within the radius of node $i$.

5. Repeat step 6 for $\mathrm{i} \leftarrow 0$ to $\mathrm{n}-1$ do

6. Select the node from the set neighbor nodes that is connected to the maximum no of nodes and make it as the dominating node of node[i].

7. End.

\subsection{Algorithm for Selecting Dominating Set in a Graph Proposed By Dai and Wu:}

Algorithm for Marking Process:

The following marking process can quickly find a strongly CDS in a given directed graph.

1. Initially assign marker $\mathrm{F}$ to each $\mathrm{u}$ in $\mathrm{V}$, where $\mathrm{V}$ is the set of all vertices of a graph.

2. Each $u$ exchanges its neighbor set $N(u)$ with all its neighbors.

Here, $u$ changes its marker $m(u)$ to $T$ if there exists vertices $v$ and w such that $(\mathrm{w}, \mathrm{u})$ and $(\mathrm{u}, \mathrm{v})$ are two edges in the graph but there exists no edges between $\mathrm{w}$ and $\mathrm{v}$.

This marking process is done by following a localized algorithm where hosts interact with neighboring host only. In the marking process, a vertex is marked $\mathrm{T}$ because it may be the only connection between its two neighbors. However, if there are multiple connections available, it is not necessary to keep all of them. We say a vertex is covered if its neighbors can reach each other via other connected marked vertices. The following dominant pruning rules are proposed and extended in to reduce the size of the CDS.

Rules for Dominating Set Reduction:

Rule1:

Consider two vertices $u$ and $v$ in $G$ '. If $N(u)-\{v\}$ is a subset of $N(v)$ in $G$, change the marker of $u$ to $F$; that is $G^{\prime}$ changes to $G^{\prime}-\{u\}$.

Rule2:

Consider two vertices $u$ and $v$ in $G^{\prime}$. If $N(u)-\{v, w\}$ is a subset of union of $\mathrm{N}(\mathrm{v})$ and $\mathrm{N}(\mathrm{w})$ in $\mathrm{G}$ then change marker of $u$ to $F$. $N(u)$ is the neighbor set of $u$ and $\mathrm{N}(\mathrm{v})$ is the neighbor set of $\mathrm{v}$.

Algorithm For Generating DA Based CDS in a Graph:

Steps:

1. Generating $\mathrm{n}$ number of nodes in random location.

2. For each node a fixed radius is set.

3. Repeat step 4 for $\mathrm{i} \leftarrow 0$ to $\mathrm{n}-1$ do

4. Now connect those node which are within the radius of node $i$.

5. Repeat step 6 for $\mathrm{i} \leftarrow 0$ to $\mathrm{n}-1$ do

6. Repeat step 7 for each sector $\mathrm{j} \leftarrow(1: \mathrm{k})$ of a node[i]

7. Select a sector of a node from the set of neighbor nodes of sector[i] that is connected to the maximum no of nodes and make it as the DS of sector[j].

8. Repeat step 10 to 11 for each node $\mathrm{i} \leftarrow 1$ to $\mathrm{n}$ do

9. if node[i] is not a dominating node Then choose only one DS with highest degree among node[i]'s chosen DS's set and remove the others. If node[i] is a dominating node and it's chosen DS's number is greater than 2 then choose the two top most dominating node with respect to highest degree.

10. If node[i] is a dominating node then check the members of node[i] with its chosen dominating nodes set. If there is no dependent member Then deselect the sectors which is chosen by its member.

11. Draw each node's chosen DSs.

12. End.

\section{COMPARISON BETWEEN RE-SULTS OF PROPOSED METH-OD AND DIE \& WU ALGORI-THM}

On comparing the outputs of program implementing Dai $\& W u$ algorithm with our own program to find out dominating set within a graph by varying the density of the graphs : we have the following results that is displayed herein.

Table 1: For 20 nodes

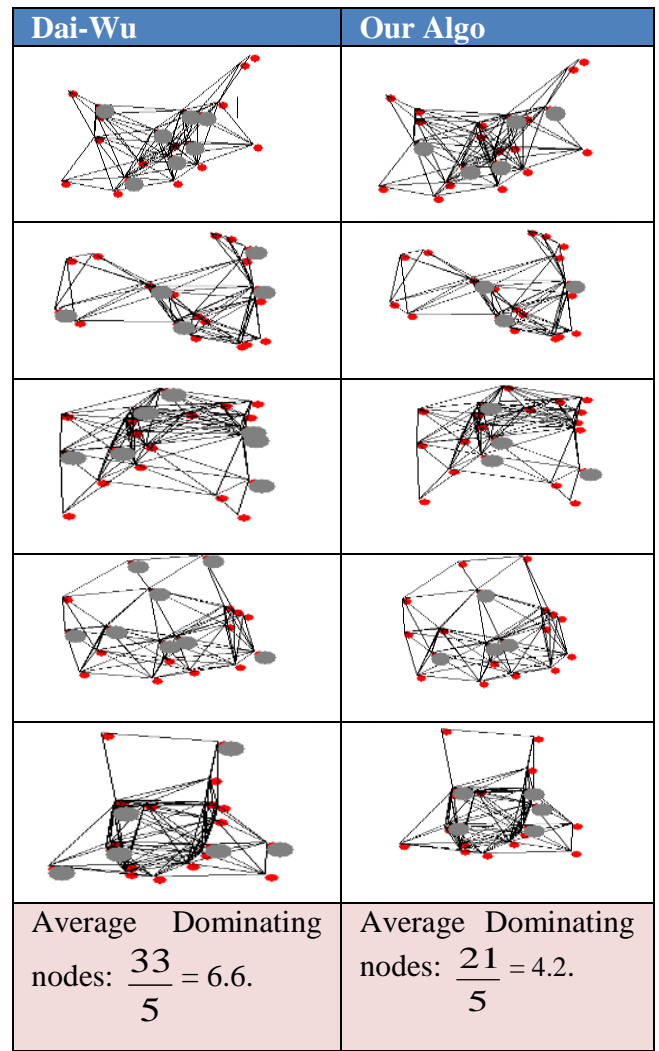

Table 2: For 30 nodes

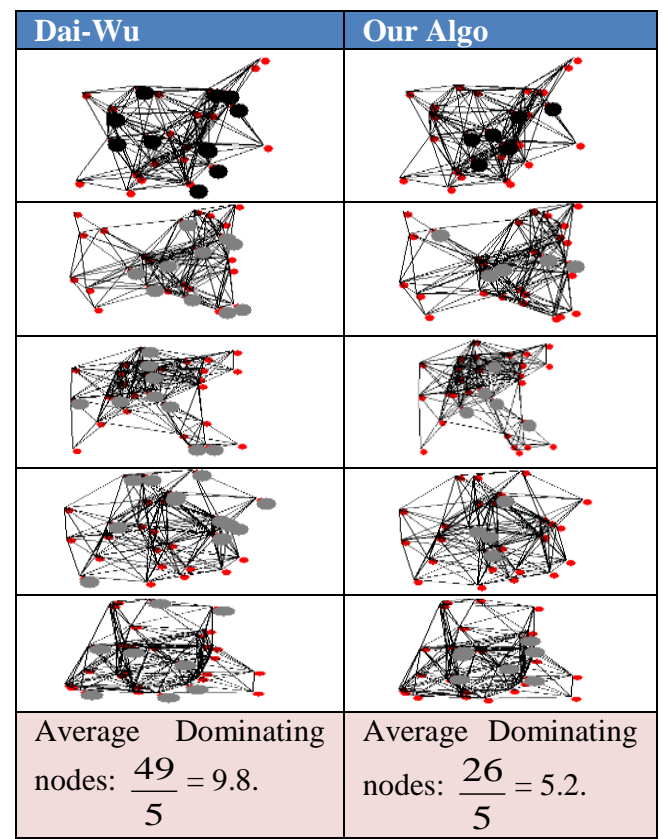


Table 3: For 40 nodes

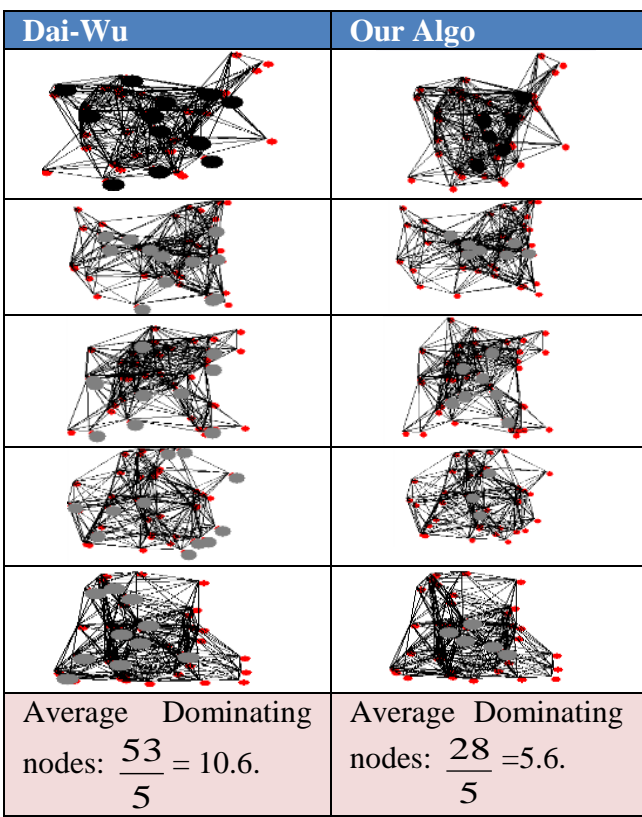

Table 4: For 50 nodes

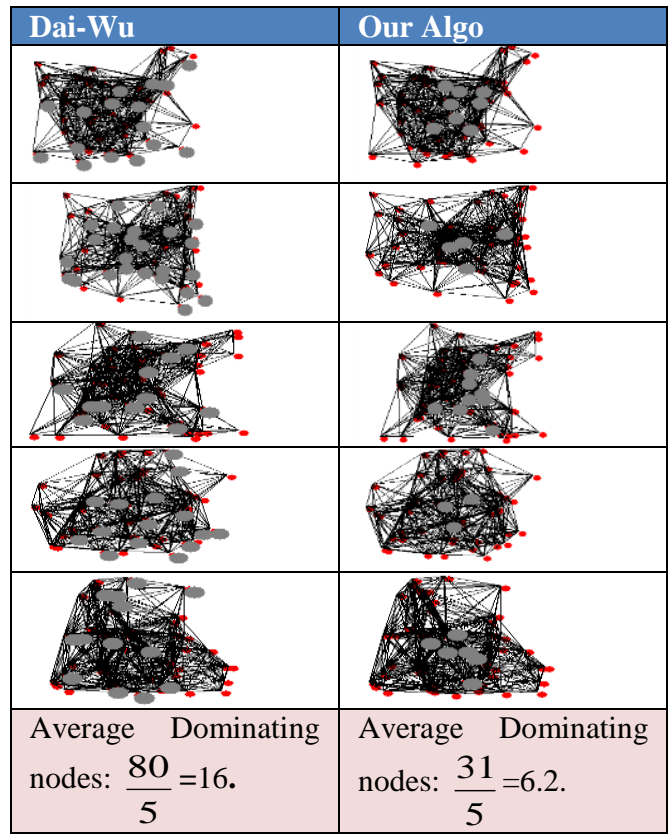

A comprehensive comparison is conducted of our implemented algorithm with the algorithm proposed by Dai-Wu. Simulation results show that our implemented algorithm works more efficiently.

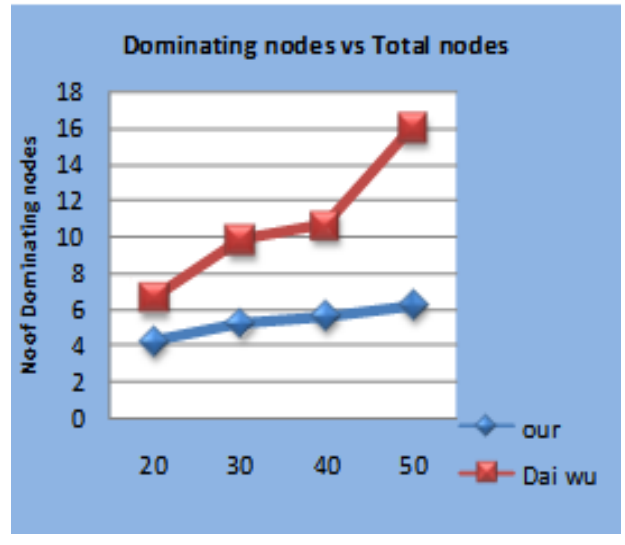

Fig 3: Comparative analysis of Output of Dai \& Wu's Algorithm of finding Dominating Nodes

The number of nodes in each network is varied and then both algorithms are used to find the connected dominating set. Next the radius of the network is varied and the same is done for each network. Results are compared and plotted in the graph. In the graph $\mathrm{X}$-axis is used to show no. of nodes in the network i.e. node density in the network and Y-axis is used to show the no of dominating nodes in the graph. It is clearly seen that our algorithm comes as the winner. No. of dominating nodes generated in our algorithm is much less than that of the Algorithm proposed by Dai-Wu. Hence it is easily verified that our algorithm works more efficiently.

The following figures shows that better energy efficiency and quality of service can be achieved using directional antenna concept.

Table 5: For 10 nodes

\begin{tabular}{|l|l|}
\hline $\begin{array}{l}\text { Dominating set } \\
\text { without using } \\
\text { concept }\end{array}$ & $\begin{array}{l}\text { Dominating set using } \\
\text { DA concept }\end{array}$ \\
\hline & \\
\hline $\begin{array}{l}\text { Average } \\
\frac{28}{3}=9.33 .\end{array}$ & DSs: \\
\hline
\end{tabular}


Table 6: For 20 nodes

\begin{tabular}{|l|l|}
\hline $\begin{array}{l}\text { Dominating set } \\
\text { without using DA } \\
\text { concept }\end{array}$ & $\begin{array}{l}\text { Dominating set using } \\
\text { DA concept }\end{array}$ \\
\hline & \\
\hline
\end{tabular}

Table 7: For 30 nodes

\begin{tabular}{|l|l|}
\hline $\begin{array}{l}\text { Dominating } \\
\text { without using } \\
\text { concept }\end{array}$ & $\begin{array}{l}\text { Dominating set using } \\
\text { DA concept }\end{array}$ \\
\hline & \\
\hline
\end{tabular}

Table 8: For 40 nodes

\begin{tabular}{|l|l|}
\hline $\begin{array}{l}\text { Dominating } \\
\text { without using } \\
\text { concept }\end{array}$ & $\begin{array}{l}\text { DA } \\
\text { DA }\end{array}$ \\
\hline
\end{tabular}

Table 9: For 50 nodes

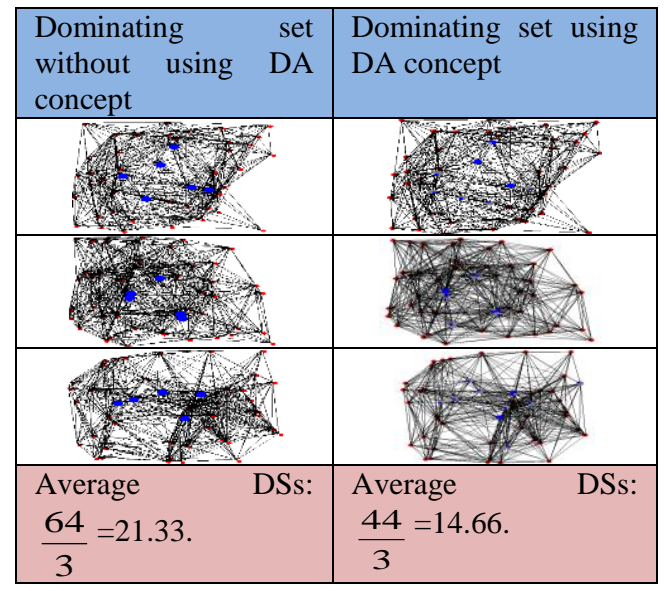

Again a comparison is made again using the concept of DS using DA model. A graph is plotted, $\mathrm{X}$-axis shows the no. of nodes in the network i.e. node density in the network and Yaxis shows the no of DSs in the graph. We assume that one node is divided into four sectors. It is clearly seen that our last algorithm which develops a sector based concept, works efficiently. No. of activated DSs generated in our last algorithm which develops sector based concept, is much less than that of the algorithm proposed for only CDS.

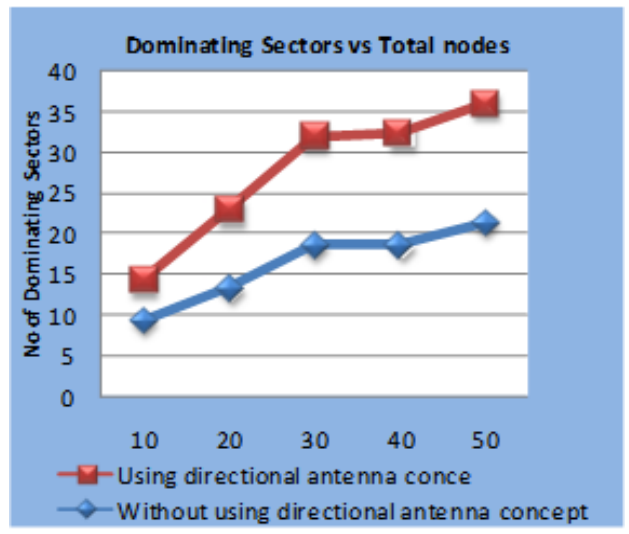

Fig 4: Comparative Analysis of Output of our algorithms one using DS without using DA concept and another using number of DSs using DA Concept.

Hence it is easily verified that our algorithm works more efficiently and Power Consumption is definitely lessens as we reduce the number of activated sectors.

\section{CONCLUSION}

In our paper, we survey the Dai \& Wu's algorithm and compare it with our own algorithm which is designed with special advantages relating to scalability, flexibility and efficient communication.

The comparison is made on the basis of following two criterions. Firstly we determined the average dominating set without varying the radius of the network. And in the next phase, we determined the minimum dominating set within the graph by varying the no of nodes only i.e. the density of the graphs.

On substituting the four sectors of each node with directional antenna, the comparison is again made between the algorithm to 
find the dominating nodes and the algorithm to find the DSs. In both cases, our proposed algorithm outshines.

Secondly, we have also made a comparative study between our own algorithm, that is, a comparison between the dominating node and DS concept. And we see that DS concepts have a lot of advantages. Some of which include that by using the sectors, transmission power is saved. All the nodes in the network choose their DS simultaneously without knowing any information about the others node. So every node acts independently to select its dominating neighbor sector. No further information is required about the others node in the network. Next, if the range covered by each node is large enough and no. of nodes in the network is large then the algorithm to find the DS provides efficiency than the algorithm to find the dominating nodes. Hence energy efficiency is achieved. Nodes do not need to be switched on every sector which is not participating in the transmission and nodes can remain silent when it has nothing to transmit to other nodes present in the network. Sectors can transmit information to all the nodes which it covers without bothering whether other sectors of that node are on or off. They act as individual directional antenna with a specific covering region. Moreover, transmission cannot be hampered due to the lack of energy. Thereby providing significant energy conservation.

\section{ACKNOWLEDGEMENT}

We express our sincere gratitude to research guide and senior faculties, Department of Computer Science and Engineering, RCC Institute of Information Technology for extending their valuable time for us to take up this problem as a paper and also financial support our institutes. This paper deals with a system that to the best of our belief does not exist as yet.

\section{REFERENCES}

[1] Shuhui Yan, Jie Wu, and Fei Dai, Efficient Directional Network Backbone Construction in Mobile Ad Hoc Networks, Department of Computer Science, Rensselaer Polytechnic Institute Troy, NY 12180.

[2] IEEE Transactions On Parallel And Distributed Systems, Vol. 19, No. 12, December 2008.

[3] F. Dai and J. Wu, “An Extended Localized Algorithm for Connected Dominating Set Formation in Ad Hoc Wireless Networks," IEEE Trans. Parallel and distributed Systems, vol. 15, no. 10, pp. 908-920, 2004.

[4] Khaled M. Alzoubi Peng-Jun Wan Ophir Frieder, New Distributed Algorithm for Connected Dominating Set in Wireless Ad Hoc Networks, Department of Computer Science, Illinois Institute of Technology, Chicago, IL 60616.

[5] S. Ikiz, V. Ogale, and V.K. Garg, "A Energy Conservation Algorithm for Mobile Ad Hoc Networks", Technical Report TR-PDS-2005-02, PDSL, ECE Dept. Univ. of Texas at Austin.

[6] A. Keshavarz-Haddad, V. Ribeiro, and R. Riedi. Broadcast capacity in multihop wireless networks. In Proc. of ACM MobiCom, 2006.

[7] R. Ramanathan. On the performance of ad hoc networks with beamforming antennas. In Proc. of ACM MobiHoc, 2001 .
[8] J. Wu. Extended dominating-set-based routing in ad hoc wireless networks with unidirectional links. IEEE Transactions on Parallel and Distributed Computing, (14):327-340, 2002.

[9] J. Wu and F. Dai. A generic distributed broadcast scheme in ad hoc wireless networks. IEEE Transactions on Computers, (10):1343-1354, 2004.

[10] F. Dai and J. Wu. Efficient broadcasting in ad hoc wireless networks using directional antennas. IEEE Transactions on Parallel and Distributed Systems, (4):1-13, 2006.

[11] An Extended Localized Algorithm for Connected Dominating Set Formation in Ad Hoc Wireless Networks by Fei Dai, Student Member, IEEE, and Jie Wu, Senior Member, IEEE.

[12] J. Wu, "Extended Dominating-Set-Based Routing in Ad Hoc Wireless Networks with Unidirectional Links," IEEE Trans. Parallel and Distributed Systems, vol. 9, no. 3, pp. 189-200, Sept. 2002.

[13] J. Wu and H. Li, "On Calculating Connected Dominating Sets for Efficient Routing in Ad Hoc Wireless Networks," Proc. Third Int'l Workshop Discrete Algorithms and Methods for Mobile Computing and Comm., pp. 7-14, 1999.

[14] C. Adjih, P. Jacquet, and L. Viennot, "Computing Connected Dominated Sets with Multipoint Relays," Technical Report 4597, INRIA-Rapport de recherche, Oct. 2002.

[15] J. Carle and D. Simplot-Ryl. Energy-efficient area monitoring for sensor networks. IEEE Computer,(2):40 46, 2004

[16] R. Ramanathan. On the performance of ad hoc networks with beamforming antennas. In Proc. of ACM MobiHoc, 2001.

[17] J. Wu and H. Li. On calculating connected dominating sets for efficient routing in ad hoc wireless networks.In Proc. of ACM DIALM'99, 1999.

[18] F. Ingelrest, D. Simplot-Ryl, and I. Stojmenovic. Optimal transmission radius for energy efficient broadcasting protocols in ad hoc and sensor networks. IEEE Transactions on Parallel and Distributed Systems,(6):536547, 2006.

[19] Antenna Orientation Optimization for Minimum-Energy Multicast Tree Construction in Wireless Ad Hoc Networks with Directional Antennas by Song Guo and Oliver Yang.

[20] J. Wu. Extended dominating-set-based routing in ad hoc wireless networks with unidirectional links.IEEE Transactions on Parallel and Distributed Computing, (14):327-340, 2002.

[21] C.C. Shen, Z. Huang, and C. Jaikaeo, "Directional Broadcast for Ad Hoc Networks with Percolation Theory," technical report, Computer and Information Sciences, Univ. of Delaware, Feb. 2004. 